\title{
The Extreme Universe Space Observatory on a Super-Pressure Balloon II Mission
}

\author{
L. Wiencke ${ }^{* a}$ and A. Olinto ${ }^{b}$ - JEM-EUSO and POEMMA Collaborations ${ }^{\dagger}$ \\ ${ }^{a}$ Colorado School of Mines \\ ${ }^{b}$ University of Chicago \\ E-mail: lwiencke@mines.edu
}

The Extreme Universe Space Observatory on a Super Pressure Balloon II Mission (EUSO-SPB2) is a precursor for a next generation space observatory for multi-messenger astrophysics. The EUSO-SPB2 instrument will measure PeV and EeV-scale cosmic rays, optical backgrounds that could mimic tau neutrino interactions in the Earth's limb, and search for optical signatures consistent with the upward-going candidate events that the Antarctic Impulse Transient Antenna (ANITA) reported. The payload, now in the design and fabrication stage, features a pair of optical telescopes that have 1 meter diameter apertures. The Fluorescence Telescope will have sensitivity in the UV to target extensive air showers at EeV energies. The Cherenkov Telescope will have UV/VIS sensitivity to target Cherenkov emission from air showers at PeV energies. The planned launch date is 2022 . We discuss the science and mission, and the current status.

36th International Cosmic Ray Conference -ICRC2019-

July 24th - August 1st, 2019

Madison, WI, U.S.A.

\footnotetext{
${ }^{*}$ Speaker.

${ }^{\dagger}$ for collaboration list see PoS(ICRC2019)1177
} 


\section{Scientific Motivation for EUSO-SPB2}

Reaching $10^{20} \mathrm{eV}(100 \mathrm{EeV})$, Ultra High Energy Cosmic rays (UHECRs) are the highest energy subatomic particles known to exist. The UHECR energy scale lies at least 5 orders of magnitude above that of the highest energy photons that have been measured from the cosmos. While the existence of UHECRs has been established for decades through measurements on the ground, their the sources and acceleration mechanisms remain unknown [1-3]. Measurements [4] by the Pierre Auger Observatory indicate that the sources are not in our galaxy.

Astrophysical PeV neutrinos, gamma rays, and UHECRs form a complementary trio of multimessenger particles from some of the most extreme energetic environments of the universe. The recent intriguing multi-messenger observations reported by IceCube and collaborating gamma ray observatories of a flaring blazar [5] open an new window in neutrino astronomy with transients.

EUSO-SPB2 is a scientific and technical sub-orbital altitude precursor for the Probe of Extreme Energy Multi-Messenger Astrophysics (POEMMA) [6]. EUSO-SPB2 also provides R\&D toward the Klypve-Extreme Universe Space Observatory (K-EUSO) [7] project. The evolution of EUSO balloon payloads is illustrated in Fig. 1.

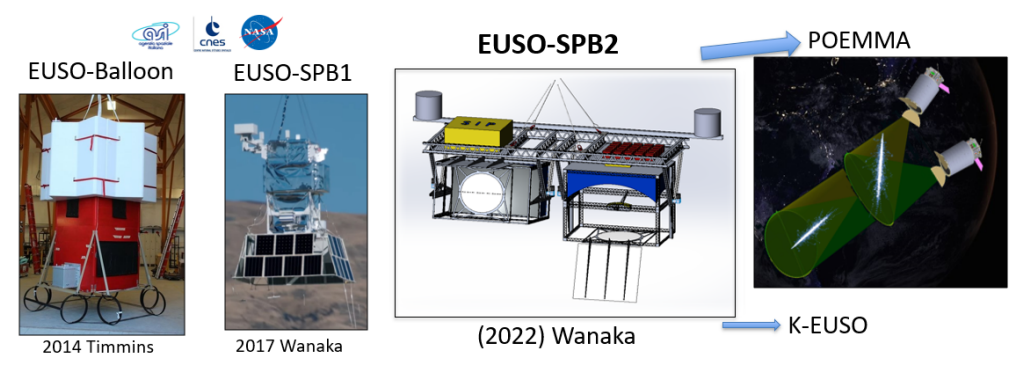

Figure 1: Evolution of the EUSO balloon payloads.

The main scientific goals of the POEMMA space mission are to discover the nature and origin of the highest energy particles in the universe [8] and to discover neutrino emission above $20 \mathrm{PeV}$ from extreme astrophysical transient sources [10]. The list of possible target sources is extensive.

POEMMA recently completed a NASA-sponsored conceptual design. Briefly, POEMMA features two identical satellites that fly in formation to make coincident measurements from the dark side of the earth of light emission from Extensive Air Showers (EASs). Each satellite is instrumented with Schmidt-based mirror optics with an entrance pupil diameter of $3.3 \mathrm{~m}$. The focal surfaces are populated with Multianode Photomultiplier Tubes (MAPMTs) across most of their area and Silicone Photomultipliers (SiPMs) across region corresponding to the "top" part of the Field of View (FOV). For UHECRs, the MAPMTs on the two satellites measure the UV nitrogen fluorescence with $1 \mu$ s time bins in stereo constrain the geometric reconstruction of the EAS direction and location. The technique leads to an estimated energy resolution of about $20 \%$ and depth of shower maximum resolution (Xmax) of 30 grams to enable measurements of UHECR energy, Xmax, and arrival direction. In turn, these parameters enable composition-enhanced anisotropy studies over the entire sky. POEMMA's sensitivity to astrophysical PeV neutrinos is maximized for earth-skimming $v_{\tau}$ s from transient sources. A PeV scale $v_{\tau}$ can interact in the earth's limb to produce a $\tau$ that exits the earth and decays in the atmosphere. The $\tau$ decay products initiate an EAS. 
The experimental signature in POEMMA is an upward-going flash of nearly parallel Cherenkov light from the EAS, observed simultaneously (after travel time correction), by both satellites in the SiPM parts of their focal surfaces. The SiPMs are digitized at $20 \mathrm{~ns}$ to increase the contrast of the fast Cherenkov flash above background. The satellites will be rotated temporarily from the nominal UHECR nadir pointing mode, to a new direction that centers the SiPM FOV on a "Target of Opportunity" transient source candidate that passes behind the earth's limb.

\section{EUSO-SPB2}

The mission, methods and science goals of EUSO-SPB2 (Fig.2) are motivated by those of POEMMA. EUSO-SPB2 will fly two EAS telescopes at $33 \mathrm{~km}$ to make the first observations at near-orbit altitude of UHECRs with the UV fluorescence technique, $\mathrm{PeV}$ cosmic rays with the direct Cherenkov technique from near-orbit altitude, and measure $v_{\tau}$ optical background signatures by looking below the earth's limb.

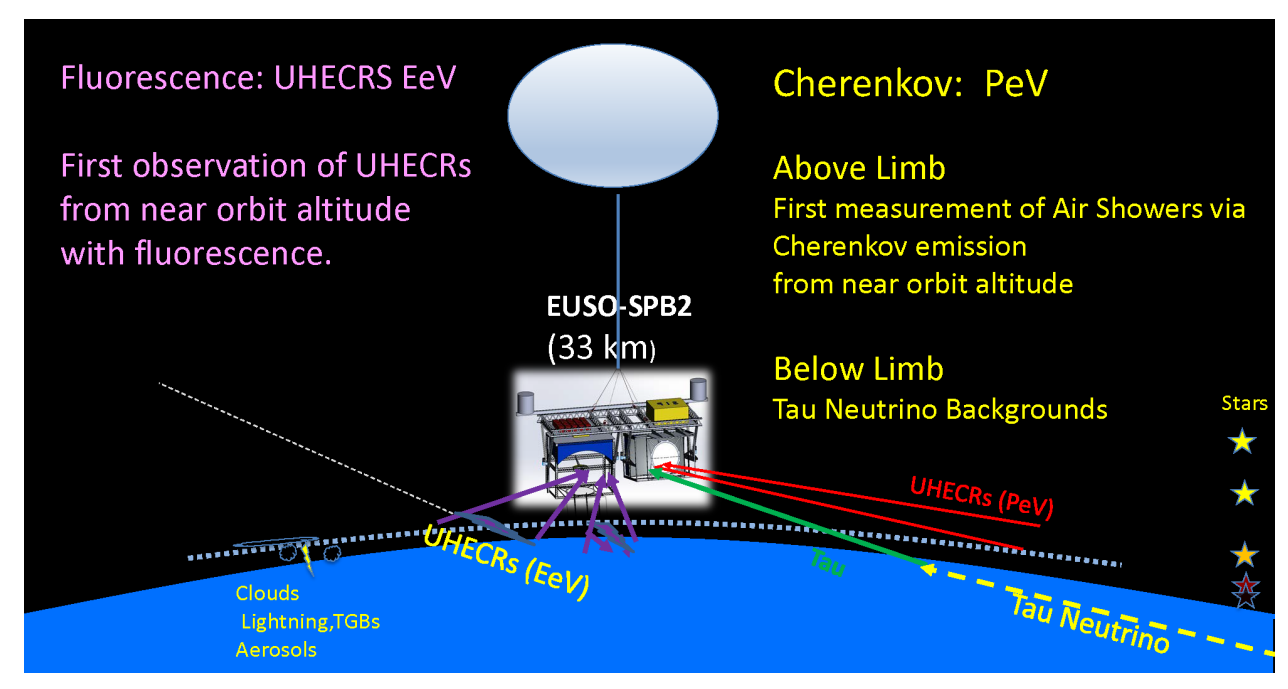

Figure 2: A conceptual view of the EUSO-SPB2 configuration at float altitude. (not drawn to scale)

Key parameters of the EUSO-SPB2 payload, telescopes, and mission are listed in Fig. 3. Like POEMMA, EUSO-SPB2 will include downward viewing for UHECRs using MAPMTs and look near the earth limb with SiPMs. For logistical and technical simplicity, the two independent telescopes will fly on one payload with one telescope, denoted as the CT, configured for specifically for PeV Cherenkov measurements and the other, denoted as the FT, configured specifically for EeV UHECR fluorescence measurements.

The EUSO-SPB2 telescope optics (Fig. 4) are a scaled down version of the POEMMA Schmidt-based optics with segmented spherical mirrors. At $1 \mathrm{~m}$ diameter, the entrance pupil area of the EUSO-SPB2 telescopes is about 1/10 that of the POEMMA telescopes. However, EUSOSPB2 will fly about 15 times closer to the ground. Like POEMMA, the imaging requirements of EUSO-SPB2 allow the optics to be designed as "light buckets", ie far from diffraction-limited. The theoretical PSF ( $80 \%$ of light spot) is about $1.5 \mathrm{~mm}$ diameter. A $3 \mathrm{~mm}$ pixel corresponds to a FOV of about $0.2^{\circ}$. The realized PSF for the 8 segment glass mirror of each telescope is expected to be $5 \mathrm{~mm}$ diameter after manufacturing and alignment precision and thermal effects. 


\begin{tabular}{|c|c|c|c|}
\hline \multicolumn{4}{|c|}{$\begin{array}{c}\text { Wanaka NZ, } 2022 \text { launch requested } \\
100 \text { days duration requested } \\
110,000 \mathrm{ft}(33 \mathrm{~km}) \text { altitude }\end{array}$} \\
\hline Telescopes & 2 & 1 Fluorescence (FT) & 1 Cherenkov (CT) \\
\hline Energy Threshold & & $\sim 3 \mathrm{EeV}$ & $\sim 10 \mathrm{PeV}$ (TBD) \\
\hline Sensor Type & & MAPMT & SiPM \\
\hline Wavelength Sensitivity & & UV $300-420 \mathrm{~nm}$ (filter) & $400-800+\mathrm{nm}$ \\
\hline Time Bin & & $1000 \mathrm{~ns}$ & $10 \mathrm{~ns}$ \\
\hline Pointing (zenith angle) & & under study & Limb $+/-10$ ? (under study) \\
\hline Field of View (available) & $40^{\circ} \times 11^{\circ}($ each $)$ & & \\
\hline FoV (instrumented) & & $\sim 35^{\circ}($ azi $) \times 11^{\circ}$ (zen) & $\operatorname{TBD}\left(16^{\circ} \times 4^{\circ} ?\right)$ \\
\hline Number of Pixels & & 6912 & 512 \\
\hline FoV (1 Pixel) & & $0.2^{\circ} \times 0.2^{\circ}$ & $0.28^{\circ} \times 0.28^{\circ}$ or $0.4^{\circ} \times 0.4^{\circ}$ \\
\hline Pixel Size & & $3 \times 3 \mathrm{~mm}$ & $4 \times 4 \mathrm{~mm}$ or $6 \times 6 \mathrm{~mm}$ \\
\hline $\begin{array}{r}\text { Optics } \\
\text { (modified Schmidt) }\end{array}$ & $\begin{array}{l}\text { Spherical Mirror, Glass } \\
\text { Radius of Curv. }=1.66 \mathrm{~m}\end{array}$ & $\begin{array}{l}8 \text { segments common focus } \\
+ \text { camera corrector/filter }\end{array}$ & $\begin{array}{l}8 \text { segments - bifocal } \\
\text { no optical filter }\end{array}$ \\
\hline Entrance Pupil & $1 \mathrm{~m}$ diameter (each) & PPMA corrector plate & PPMA corrector plate \\
\hline Payload Weight & $\sim 2500$ lbs (1000 gondola) & $\sim 750 \mathrm{lbs}$ & $\sim 750 \mathrm{lbs}$ \\
\hline \multicolumn{4}{|c|}{ Earth Limb at Float: $5.8^{\circ}$ below horizontal, $649 \mathrm{~km}$ from payload } \\
\hline
\end{tabular}

Figure 3: Selected parameters of the EUSO-SPB2 mission and instrument
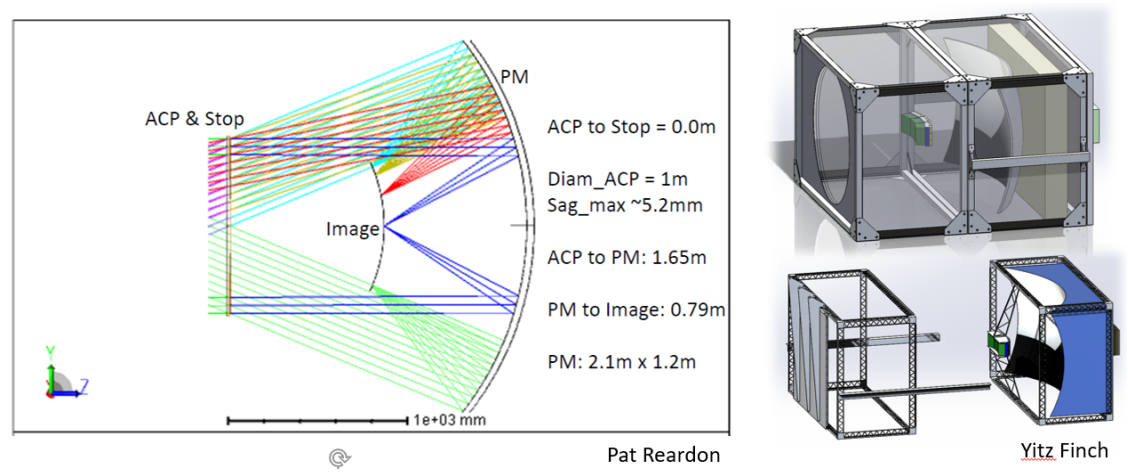

Figure 4: The optical design of EUSO-SPB2 (left panel) PM (Primary Mirror) APC (Aperture Corrector Plate) The telescope structure and camera location are shown in the right panel.

The Fluorescence telescope camera builds on the designs flown in the 2014 overnight EUSOBalloon Mission that made the first observation of speed of light (laser) tracks by looking down [11], the 2017 EUSO-SPB1 mission of opportunity [12,13] that recorded optical backgrounds, instrument data and low energy cosmic rays incident on the camera, but terminated prematurely in the Pacific Ocean, the EUSO-TA ground prototype [14] and the MINI-EUSO instrument [15] planned for launch to the ISS in 2019. The EUSO-SPB2 FT camera features 256 pixel elementary cells (ECs) comprised of 4 MAPMTs (Fig. 5). These ECs include signal integration to obtain photoelectron counts. The camera will include 27 ECs for a total of 6912 pixels. One design challenge is accommodating the flat EC units to the curved image surface. A small corrector lens will be placed in front of each $3 \times 3 \mathrm{EC}$ array to preserve the PSF.

For the FT pointed in nadir mode, the EAS trigger rate currently estimated to be about $0.2 / \mathrm{hr}$ with a trigger threshold of about $1 \mathrm{EeV}$. A $30^{\circ}$ tilt angle below horizontal yields a $0.09 / \mathrm{hr}$ trigger 

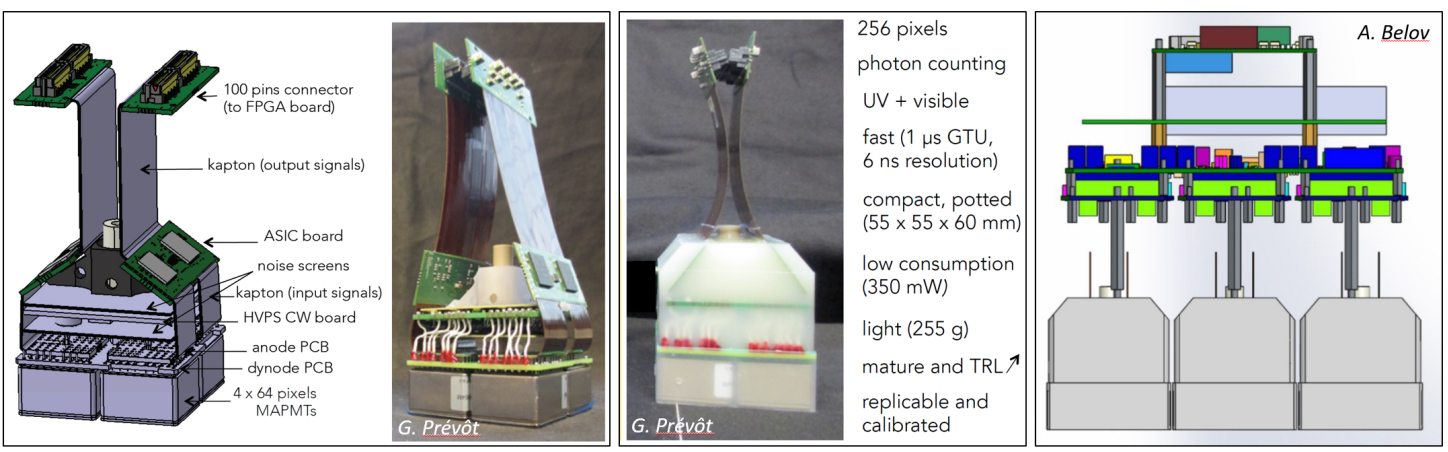

Figure 5: The $2 \times 2$ multi-anode photomultiplier assembly (256 channels) with integrated signal digization in photoelectron counts. Left: Design and prototype. Center: Prototype after potting. Right: Side view of configuration of 2304 pixel array with trigger/event builder electronics and HV controls.

rate with $10 \mathrm{EeV}$ threshold. Examples of simulated EASs for which Xmax falls in the FT FOV are shown in Figs 6.
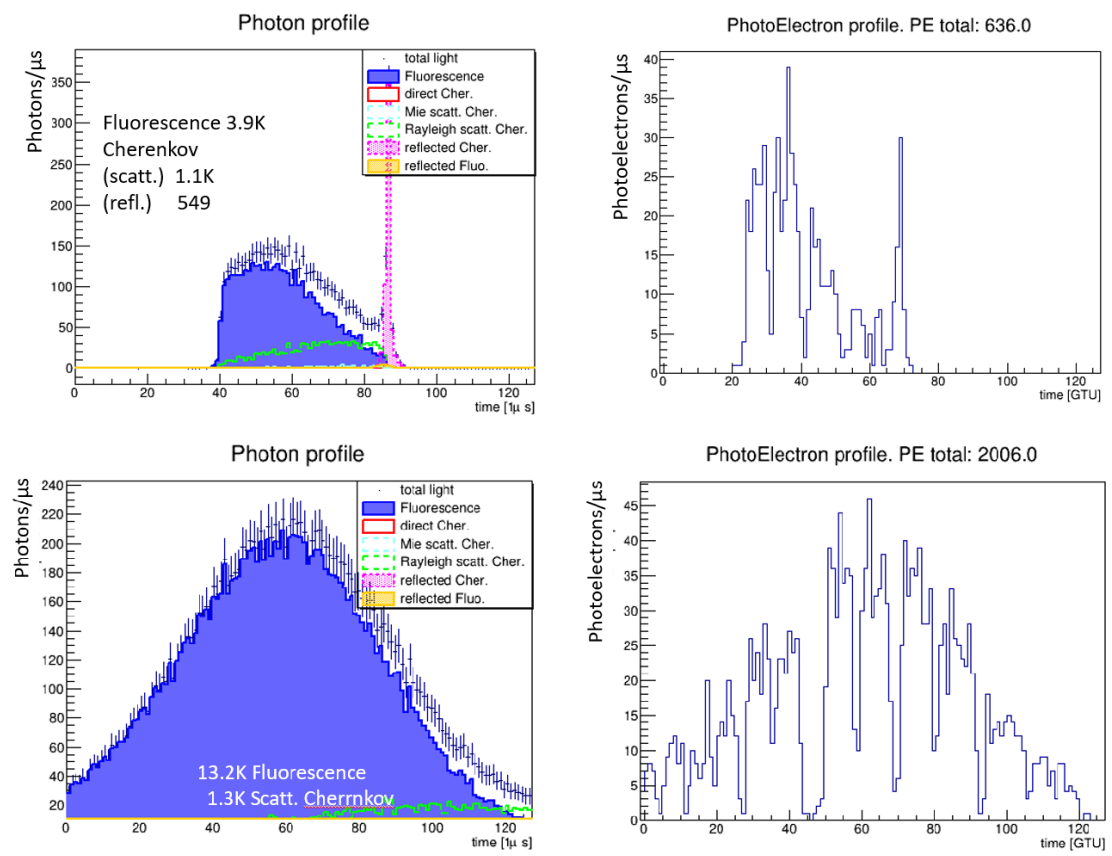

Figure 6: Simulated extensive air shower as recorded by EUSO-SPB2. Upper Left: a $3 \mathrm{EeV}$ proton, $34^{\circ}$ zenith angle. Light curve at telescope pupil with the FD tilted to nadir. Upper Right: Corresponding photoelectron counts, $1 \mu$ s time bin. Lower Left: a $10 \mathrm{EeV}$ proton, $67^{\circ}$ zenith angle. Light curve at telescope pupil with FD tilted $30^{\circ}$ below horizontal. Lower Right: Corresponding photoelectron counts, $1 \mu$ s time bin.

The FT will also house a dual-band IR Camera camera system [19] designed to monitor the presence of clouds and measure cloud-top temperatures.

A preliminary simulation of an expected Cherenkov signal [17] signature of a Earth-skimming $v_{\tau}$ as seen from $33 \mathrm{~km}$ altitude is shown in Fig. 7. Further simulations are in progress [17].

To provide a coincidence measurement of Cherenkov light spots from EASs, the CT mirror 
segments will be aligned in a bi-focal configuration. The bi-focal alignment is realized by rotating the upper 4 segments and the lower 4 segments relative to each other by a few PSF equivalents in azimuth. A parallel light pulse from outside the telescope will produce two lights spots on the focal surface and thus distinguish this trigger of interest from a single cluster of triggered pixels caused by a low energy cosmic primary striking the SiPM array. Such triggers from direct cosmics were observed in the EUSO-SPB 1 camera at float altitude. The Cherenkov Telescope focal surface will be populated by 512 SiPMs. The final selection is being finalized, as is their arrangement on the image surface. Reference [16] details the camera development and status.
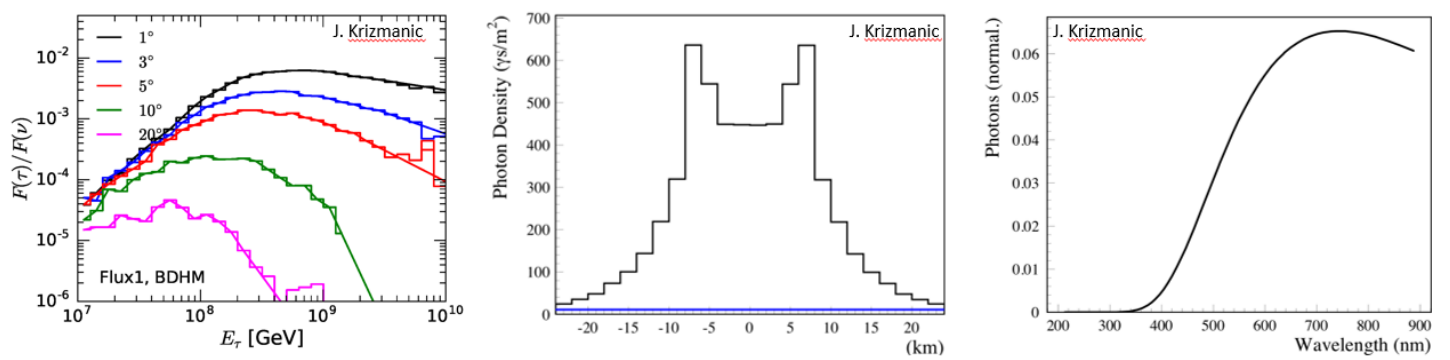

Figure 7: Preliminary simulations of Earth skimming $v_{\tau}$ signatures for the $33 \mathrm{~km}$ altitude of EUSO-SPB2. Left: Ratio of tau emerging flux vs incident neutrino flux for different Earth emergence angles. Center: Cherenkov light distribution for a 100 PeV EAS 5 deg Earth emergence angle. The two peaks are separated by $3 \mathrm{deg}$ at $33 \mathrm{~km}$. Right: The corresponding Cherenkov light spectrum.

Airglow MOMitor (AMON) and EUSO telescope darkness MONitor (EMON) detectors will be used as ancillary instruments. AMON is a one pixel instrument [20] that will measure the absolute intensities of the radiation from the limb and the atmosphere below. Two AMON instruments will operate in the same wavelength bands of the CT and FT to monitor airglow and night atmosphere radiation backgrounds. The EMON instruments will be located inside the CT and FT telescopes to monitor darkness conditions during operation and testing.

Extensive laboratory and field testing is planned to characterize and calibrated the CT and FT prior to payload integration. Their PSFs and optical throughput will be measured for downward and horizontal orientations using a $1 \mathrm{~m}$ diameter optical test beam. The CT and FT will be transported to the Utah desert or a higher elevation location for testing with lasers and other light sources, building on the EUSO-SPB1 field campaign [18]. This campaign will also include observation time to record EASs via Fluorescence and Cherenkov light.

\section{Mission}

A launch from NASA's Wanaka facility on a super pressure balloon is motivated by the opportunity for flight of up to 100 days. The $45^{\circ}$ latitude, allows the FT and CT to collect data at night while the sky is dark. During the day solar panels recharge the batteries that power the instrument and other systems, including those for safe termination over land. NASA has been granted balloon over-flight and landing permissions routinely by the countries along this southern latitude.

Wanaka lies under a stratospheric air circulation that forms each Fall and Spring. During the Southern Fall, this wide river of high thin air circles the Southern globe to the east at 50-150 
$\mathrm{km} / \mathrm{h}$. A March/April launch window can insert the balloon into this circulation. The balloon and telescopes travel together in an apparent wind speed of essentially zero. The super pressure balloon is sealed. At float it expands to a maximum constrained volume of 18 million $f t^{3}$ with a slight overpressure that remains positive at night to maintain the fixed volume. Thus the balloon floats at a nearly constant altitude. Absent technical issues, an SPB can remain aloft almost indefinitely.

To support the mission, the EUSO-SPB2 gondola (preliminary design: (Fig. 8) houses the CT, FT, the data and command telemetry systems, various antennas, a CPU, instrument monitoring systems, and two solar power systems. The gondola also houses systems for monitoring and operating the balloon and related systems, including ballast hoppers and flight termination control hardware, which are the responsibility of personnel from NASA's Columbia Scientific Balloon Facility.

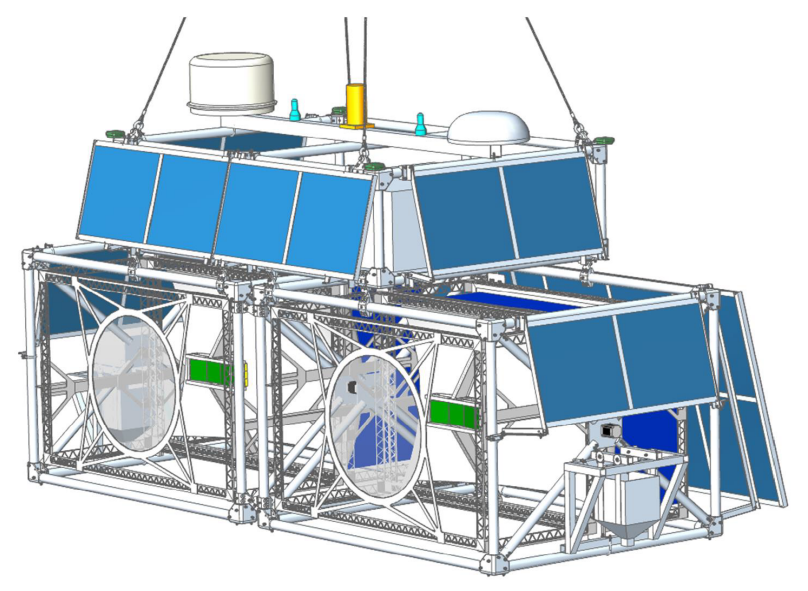

Figure 8: The EUSO-SPB2 payload including gondola structure shown in a premininary configuration, telescopes, solar panels, antennas, and ballast hoppers.

The mission will focus on data collection during dark periods when the moon is down for the best contrast for the optical signatures of scientific interest. The instrument solar panels will be mounted on the opposite side of the telescopes' apertures. A azimuth rotator with a sun tracker will turn the gondola so that this solar array tracks the sun. At night, the rotator will use feedback from a differential GPS system to control the azimuth direction. Nightly data taking will start with the telescopes opposite sunset in azimuth to catch the earliest dark backgrounds. Conversely, the nightly operation will terminate with the solar panels pointed toward the next sunrise. Instrument operations and monitoring will be shared between collaborators in the US, Japan and Europe to provide, during local daytime, control and monitoring of the telescopes 24/7.

The CT will spend some observational time pointed above the $-5.8^{\circ}$ limb angle to record direct Cherenkov measurements from $\mathrm{PeV}$ cosmic rays. In addition to their first observation from nearorbit altitude, these events are expected to provide insitu estimates of the optics PSF and test the bifocal double-spot triggering algorithm. The bulk of the observing time will be devoted to measurements below the limb with the CT pointed in the directions from which upward tau-induced EASs are expected to originate. The zenith pointing direction(s) of the FT during the mission will be finalized once the simulations studies have been completed. Searches for ANITA event-like candidates are under discussion.

This work was partially supported by NASA awards 11-APRA-0058, 80NSC18K0477, 
80NSSC19K0627 in the USA, Basic Science Interdisciplinary Research Projects of RIKEN and JSPS KAKENHI Grant (JP17H02905, JP16H02426 and JP16H16737), by the Italian Ministry of Foreign Affairs and International Cooperation, by the Italian Space Agency through the ASI INFN agreement n. 2017-8-H.0 and contract n. 2016-1-U.0, by by the French space agency CNES, by Slovak Academy of Sciences MVTS JEMEUSO and VEGA grant agency project 2/0132/17, by National Science Centre in Poland grant, 2017/27/B/ST9/02162) Russia is supported by ROSCOSMOS and the Russian Foundation for Basic Research Grant No 16-29-13065. Sweden is funded by the Olle Engkvist Byggmästare Foundation.

\section{References}

[1] K. Kotera and A. V. Olinto, The astrophysics of ultrahigh energy cosmic rays, Ann. Rev. Astron. Astrophys. 49, 119 (2011) doi:10.1146/annurev-astro-081710-102620 arXiv:1101.4256.

[2] R. A. Batista et al., Open Questions in Cosmic-Ray Research at Ultrahigh Energies, Frontiers in Astronomy and Space Sciences 2019.

[3] L. A. Anchordoqui, Ultra-high-energy cosmic rays, (2019) arXiv:1807.09645 [astro-ph.HE].

[4] A. Aab et al. [The Pierre Auger Collaboration] Observation of a large-scale anisotropy in the arrival directions of cosmic rays above $8 \times 1018 \mathrm{eV}$, Science 357 (2017). DOI: 10.1126/science.aan4338

[5] M. G. Aartsen et al. [IceCube Collaboration], Multimessenger observations of a flaring blazar incident with high-energy neutrino IceCube-170922A, Science 361, (2018), arXiv:1807.08816.

[6] A. Olinto et al. POEMMA (Probe Of Extreme Multi-Messenger Astrophysics) design, (2019), arXiv:1907.06217.

[7] M. Casolino et al. [JEM-EUSO Collaboration], KLYPVE-EUSO: Science and UHECR observational capabilities, PoS ICRC 2017368 (2018).

[8] L. A. Anchordoqui et al., UHECRs with POEMMA, (2019) arXiv:1907.03694.

[9] P. Meszaros, et al. Astrophysical Sources of High Energy Neutrinos in the IceCube Era, Ann. Rev. Nucl. Part. Sci. 67, (2017), 45-67; arXiv:1708.03577.

[10] T. M. Venters, et al., POEMMA's Target of Opportunity Sensitivity to Cosmic Neutrino Transient Sources, (2019). arXiv:1906.07209

[11] G. Abdellaoui et al. [JEM-EUSO Collaboration], First observations of speed of light tracks by a fluorescence detector looking down on the atmosphere, JINST,13, (2018).

[12] L. Wiencke et al. [JEM-EUSO Collb.], EUSO-SPBI mission and science, PoS ICRC 20171097 (2018).

[13] J. Eser et al., [JEM-EUSO Collaboration], Results of the EUSO-SPB1 flight, POS Proc. ICRC (2019).

[14] G. Abdellauoi et al. [JEM-EUSO Collaboration], EUSO-TA - First results from a ground-based EUSO telescope, Astroparticle Physics, 102, 98 (2018).

[15] F. Capel et al. [JEM-EUSO Coll.], Mini-EUSO: A high resolution detector for the study of terrestrial and cosmic UV emission from the International Space Station, Adv. SR, 62 (2018).

[16] N. Otte, E Gazda, E. Judd, J. Kutzenzov, O. Romero Matamala, P. Reardon and L. Wiencke, Development of a Cherenkov Telescope for the Detection of Ultra-High Energy Neutrinos with EUSO-SPB2 and POEMMA, POS Proc. ICRC 2019977.

[17] J. Krizmanic et al., nuSpaceSim: A Comprehensive Neutrino SimulationPackage for Space-based \& Suborbital Experiments, PoS Proc. ICRC 2019936.

[18] J. Eser et al., [JEM-EUSO Coll.], Preflight calibration and testing of EUSO-SPB1 in the lab and the desert in Proc 35th ICRC, (Busan), PoS ICRC 2017457 (2017).

[19] R. Diesing et al., IR Cloud Camera System for EUSO-SPB2, POS Proc. ICRC 2019241.

[20] S. Mackovjak, et al., Airglow monitor by one-pixel detector, NIMA 922, 150, (2019). 